\title{
Aging increases oxidative stress and renal expression of oxidant and antioxidant enzymes that are associated with an increased trend in systolic blood pressure
}

Pedro Gomes, Sónia Simão, Elisabete Silva, Vanda Pinto, João S. Amaral, Joana Afonso, Maria Paula Serrão, Maria João Pinho and Patrício Soares-da-Silva*

Institute of Pharmacology and Therapeutics; Faculty of Medicine; University of Porto; Porto, Portugal

Abbreviations: DTT, dithiothreitol; EDTA, ethylene diamine tetra-acetic acid; GAPDH, glyceraldehyde-3-phosphate dehydrogenase; GPx, glutathione peroxidase; $\mathrm{H}_{2} \mathrm{O}_{2}$, hydrogen peroxide; HEPES, 4-(2-hydroxyethyl)-1-piperazine ethanesulfonic acid; MDA, malondialdehyde; NADPH, nicotinamide adenine dinucleotide phosphate; Nox, NADPH oxidase; ROS, reactive oxygen species; SDS-PAGE, sodium dodecyl sulfate polyacrylamide gel electrophoresis; SHR, spontaneously hypertensive rat; SOD, superoxide dismutase; TBA, thiobarbituric acid; TCA, trichloroacetic acid; WKY, Wistar Kyoto

Key words: aging, oxidative stress, kidney, hydrogen peroxide, NADPH oxidase, antioxidant enzymes, Wistar Kyoto rat

The aim of this study was to investigate whether the effects of aging on oxidative stress markers and expression of major oxidant and antioxidant enzymes associate with impairment of renal function and increases in blood pressure. To explore this, we determined age-associated changes in lipid peroxidation (urinary malondialdehyde), plasma and urinary hydrogen peroxide $\left(\mathrm{H}_{2} \mathrm{O}_{2}\right)$ levels, as well as renal $\mathrm{H}_{2} \mathrm{O}_{2}$ production, and the expression of oxidant and antioxidant enzymes in young (13 weeks) and old ( 52 weeks) male Wistar Kyoto (WKY) rats. Urinary lipid peroxidation levels and $\mathrm{H}_{2} \mathrm{O}_{2}$ production by the renal cortex and medulla of old rats were higher than their young counterparts. This was accompanied by overexpression of NADPH oxidase components Nox4 and p22 phox in the renal cortex of old rats. Similarly, expression of superoxide dismutase (SOD) isoforms 2 and 3 and catalase were increased in the renal cortex from old rats. Renal function parameters (creatinine clearance and fractional excretion of sodium), diastolic blood pressure and heart rate were not affected by aging, although slight increases in systolic blood pressure were observed during this 52-week period. It is concluded that overexpression of renal Nox4 and p22phox and the increases in renal $\mathrm{H}_{2} \mathrm{O}_{2}$ levels in aged WKY does not associate with renal functional impairment

*Correspondence to: Patrício Soares-da-Silva; Institute of Pharmacology and Therapeutics; Faculty of Medicine; University of Porto; Alameda Prof. Hernâni Monteiro; Porto 4200-319 Portugal; Tel.: +351.225.513.642; Fax:+351.225.513.643; Email: pss@med.up.pt

Submitted: 03/04/09; Revised: 04/22/09; Accepted: 04/22/09

Previously published online as an Oxidative Medicine and Cellular Longevity E-publication:

http://www.landesbioscience.com/journals/oximed/article/8819 or marked increases in blood pressure. It is hypothesized that lack of oxidative stress-associated effects in aged WKY rats may result from increases in antioxidant defenses that counteract the damaging effects of $\mathrm{H}_{2} \mathrm{O}_{2}$.

\section{Introduction}

Aging is a biological process accompanied by gradual deterioration of the physiological functions and metabolic processes. This multifactorial process, which is affected by the sum of genetic and environmental factors, differently encompasses various organs and tissues. There is increasing evidence for an age-related decline in renal function, both in animal models and in humans. ${ }^{1-6}$ Several studies show this function decline to be associated with both structural (glomerulosclerosis, tubular atrophy and interstitial fibrosis) and functional [decreases in glomerular filtration rate (GFR), proteinuria, reduced ability to concentrate or dilute urine, impairment of electrolyte and ion transport, alteration in hormonal functions, reduced drug excretion] changes in the kidney (reviewed in refs. 5 and 6). The molecular mechanisms and the cellular changes underlying the functional and structural changes associated with the renal aging are currently under active investigation. In general, the theories of cellular senescence include genomic instability and telomere loss, oxidative damage, genetic programming and cell death. ${ }^{7}$ Among these, the oxidative stress theory of aging states that declines in organism function, that characterize the aging process, result from a progressive accrual of oxidative damage to cellular constituents. ${ }^{8,9}$ ROS produced as a by-product of normal aerobic metabolism continuously damage cellular nucleic acids, proteins and membrane lipids. ${ }^{10}$ Various biomarkers of oxidative stress such as oxo-2-deoxyguanosine, ${ }^{11} \mathrm{H}_{2} \mathrm{O}_{2},{ }^{12}$ 3-nitrotyrosine (3-NT), ${ }^{13} \mathrm{MDA},{ }^{14}$ and advanced glycation end products (AGEs) ${ }^{15}$ increase with age in renal tissues. The extent of 
cellular damage and aging rely on the balance between production of oxidants and the removal by the antioxidant system.

Excess oxidants are captured by SOD, GPx and catalase. Glutathione-S-transferase (GST) catalyzes the nucleophilic addition of the thiol of GST to electrophilic acceptors. Several lines of evidence support the view that increased longevity without decreases in renal function is associated with increases in expression of antioxidant enzymes. ${ }^{16-18}$

The rat provides a useful experimental model to study the mechanisms of kidney aging. As in man, a wide diversity in the renal response to aging occurs in the rat. Most rat models of aging display chronic progressive nephrosis, although the rate at which injury develops is highly variable. On the other hand, comparisons of rats with different genetic backgrounds will allow differentiation between the impact of disease processes such as cancer, atherosclerotic disease and inflammatory disorders, from the aging process.

The characterization of oxidative stress in the aging WKY, a normotensive rat strain, has not been reported before. Therefore, the major goal of the present study was to evaluate the antioxidant/ pro-oxidant status in the aging WKY. In addition, the effect of age on renal function, blood pressure and metabolic profile was also investigated in WKY.

\section{Results}

General data. Body weight increased steadily until 15-20 weeks of age (Fig. 1A). After this period, the weight of the animals continued to increase, but at a slower rate (Fig. 1A and Table 1). Tibial length is an index of growth, which remains constant after maturity. As shown in Table 1, tibial length was slightly increased in old rats, indicating that young (13-week-old) rats were reaching full body growth. In contrast, body weight/tibial length ratio increased approximately two-fold in the old animals, suggesting that aged WKY rats accumulate fat mass. The diastolic blood pressure and heart rate remained virtually unchanged throughout the study period, though slight increases in systolic blood pressure were observed at weeks 32 and 36 of age (Fig. 1B and C and Table 1). Average water intake did not differ between groups, but food intake was reduced in the old animals. Urinary protein excretion, fractional urinary excretion of sodium $\left(\mathrm{FE} \mathrm{Na} \mathrm{Na}^{+}\right.$) and creatinine clearance, commonly used markers of renal function, were not different between young and old rats (Table 1), indicating that the renal function was preserved in the old animals.

Metabolic parameters. To investigate the effect of age on some features of the metabolic profile, the mean non-fasting plasma glucose, cholesterol and triglyceride concentrations were measured in young and old rats. We found that the metabolic parameters were not significantly different between young and old rats (glucose: $141.5 \pm 10.1$ vs. $141.2 \pm 5.3 \mathrm{mg} / \mathrm{dl}$; total cholesterol: $78.2 \pm 3.6$ vs. $82.1 \pm 6.8 \mathrm{mg} / \mathrm{dl}$; triglycerides: $82.2 \pm 8.6$ vs. $86.6 \pm 7.5 \mathrm{mg} / \mathrm{dl})$.

Biomarkers of oxidative stress. A rapidly growing body of evidence indicates that oxidative stress plays a role in the aging process. ${ }^{19,20}$ Therefore, we addressed the question of whether oxidative stress is increased in old rats. For that purpose, we determined the plasma and urinary levels of $\mathrm{H}_{2} \mathrm{O}_{2}$, a hazardous ROS against tissues and cells, as a marker of systemic oxidative stress. No significant change in urinary levels of $\mathrm{H}_{2} \mathrm{O}_{2}$ was

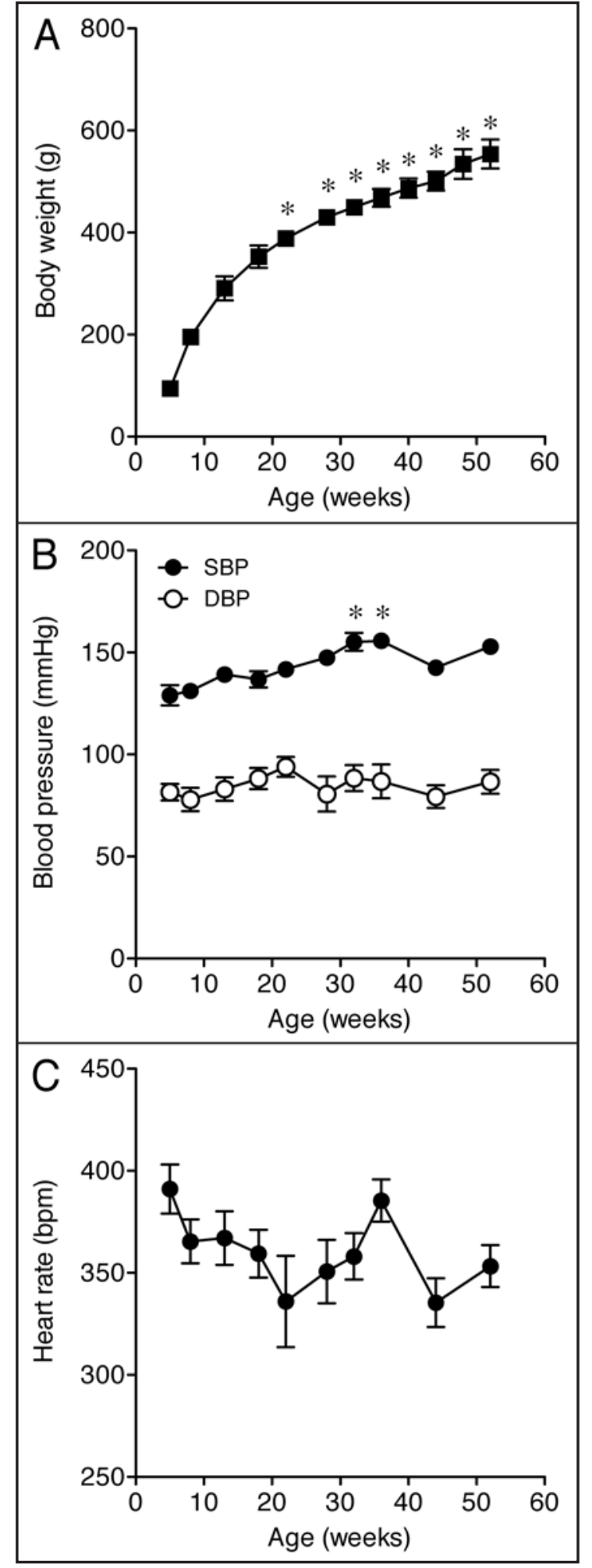

Figure 1. Changes in (A) body weight, (B) systolic and diastolic blood pressure and $(C)$ heart rate of Wistar Kyoto (WKY) rats as a function of age. Each point represents the mean \pm SEM of 6-12 rats. Significantly different from values in 13 -week-old rats $\left({ }^{*} p<0.05\right)$ using the Dunnett multiple comparison test. 
Table 1 Physiological parameters

\begin{tabular}{lcc} 
Parameter & Young & Old \\
Body weight $(\mathrm{g})$ & $311 \pm 4$ & $571 \pm 15^{*}$ \\
Tibial length $(\mathrm{cm})$ & $4.01 \pm 0.09$ & $4.27 \pm 0.03$ \\
Body weight/tibial length $(\mathrm{g} / \mathrm{cm})$ & $69.1 \pm 2.1$ & $131.9 \pm 2.7^{*}$ \\
Systolic blood pressure $(\mathrm{mm} \mathrm{Hg})$ & $139 \pm 3$ & $153 \pm 1$ \\
Diastolic blood pressure $(\mathrm{mm} \mathrm{Hg})$ & $83 \pm 6$ & $87 \pm 6$ \\
Heart rate (beats/min) & $367 \pm 13$ & $353 \pm 10$ \\
Proteinuria $(\mathrm{mg} / 24 \mathrm{~h})$ & $14.6 \pm 1.2$ & $14.3 \pm 0.9$ \\
FE Na+ $\%)$ & $0.38 \pm 0.08$ & $0.31 \pm 0.03$ \\
Creatinine clearance $(\mathrm{ml} / \mathrm{min})$ & $2.42 \pm 0.42$ & $2.99 \pm 0.19$ \\
\hline
\end{tabular}

${ }^{*} p<0.05$ compared with young rats.

observed between young and old rats (Fig. 2A), although a slight trend towards increased plasma $\mathrm{H}_{2} \mathrm{O}_{2}$ levels was detected in old rats (Fig. 2B). We next measured the concentration of urinary MDA, classified as a marker of lipid peroxidation. ${ }^{14}$ As shown in Figure $2 \mathrm{C}$, the levels of MDA in urine were significantly increased at 52 weeks of age.

Because elevated urinary MDA excretion might reflect an increase in oxidative stress in the kidney, ${ }^{21}$ we assessed $\mathrm{H}_{2} \mathrm{O}_{2}$ production by renal tissue. The local production of $\mathrm{H}_{2} \mathrm{O}_{2}$ by both kidney cortex and medulla was significantly higher in old than in young WKY (Fig. 2D and E). Altogether, these results indicate the occurrence of oxidative stress in the renal tissue during aging in the normotensive WKY strain.

NADPH oxidase subunits. The age-related increases in oxidative stress levels and oxidative damage to biomolecules can be the result of an increased production of reactive oxygen species or diminished antioxidant defenses, or both. ${ }^{8,9}$ The protein expression of some components of the pro-oxidant NADPH oxidase enzyme complex was analyzed in kidney cortex extracts. As depicted in Figure 3, protein levels of NADPH oxidase membrane-associated components, Nox4 and p22phox, were significantly upregulated in old as compared to young WKY.

Antioxidant enzymes. Having determined an upregulation of the pro-oxidant enzyme NADPH oxidase, we next investigated changes in expression of the major antioxidant enzymes. Protein abundance of SOD isoforms (cytoplasmic SOD1, mitochondrial SOD2 and extracellular SOD3) was evaluated in the renal cortex of young and old WKY. Protein levels of both SOD2 and SOD3 isoforms were significantly increased in the renal cortex of old rats, whereas SOD1 remained unaltered during aging (Fig. 4). To further analyze antioxidant enzyme expression in the aged kidney, we measured catalase and GPx, which detoxify $\mathrm{H}_{2} \mathrm{O}_{2}$, the product of SOD action. A marked increase in immunodetectable catalase was found in the renal cortex of old rats, while GPx expression remained constant over the study duration (Fig. 5).

\section{Discussion}

The motivation for this study was to characterize the aging process in the WKY rat focusing on oxidative stress status in the kidney. We found that until 52 weeks of age no significant changes were observed in renal function, blood pressure and metabolic parameters of WKY. However, renal biomarkers of oxidative stress increased markedly with aging. Additionally, NADPH oxidase membrane components were upregulated and antioxidant systems were shown to be activated in old WKY.

During aging of WKY there is a marked increase in the ratio of body weight to tibia length due to fat mass accumulation, this being particularly evident in the retroperitoneal white adipose tissue (data not shown). In agreement with other studies, ${ }^{22-28}$ blood pressure as well as heart rate remained almost unaltered during aging, with the exception of a slight, but statistically significant, increase in systolic blood pressure at weeks 32 and 36 of age. Similar to our findings, Susic et al. ${ }^{29}$ have reported that WKY develop isolated systolic hypertension (ISH) with advancing age. ISH is the predominant form of hypertension in the elderly population ${ }^{30}$ and is associated with an increased risk of cardiovascular diseases, especially stroke and cardiovascular death. ${ }^{31,32}$ It is thought that ISH is caused mainly by large artery stiffening, resulting from disruption and fatigue-fracture of elastic fibers. However, the precise mechanisms underlying this age-associated effect are currently unknown and beyond the scope of the present study.

Metabolic parameters, in particular, non-fasting plasma glucose, total cholesterol and triglyceride concentrations, remained practically unchanged until 52 weeks of age.

Other studies support our findings and reported no differences in glucose and lipid parameters between young and old WKY. 24,26,27 Taken together, these results suggest that the normotensive WKY strain develops obesity with aging, with no evidence of metabolic abnormalities.

The kidney is one of the organs with higher susceptibility to the development of age dependent tissue damage ${ }^{1,27}$ and male rats most frequently die of renal failure. ${ }^{18,33}$ It is also known that elderly human subjects exhibit reduced renal function characterized by decreases in GFR, a decline in the ability to produce a concentrated urine, impaired tubular reabsorption and water homeostasis and albuminuria. ${ }^{34}$ The resulting deterioration in renal function limits general health and life expectancy and in turn may impact on cardiovascular and metabolic diseases. However, none of the parameters used to evaluate kidney function were altered with aging in male WKY rats, demonstrating that renal function was apparently well preserved up to 52 weeks of age. One possible explanation for the discrepancy between our results and those of Percy et al. ${ }^{27}$ may have resulted from differences in age between animals used in each study. Another potential reason for the absence of renal dysfunction in 52-week-old WKY may be related to the parameters used to monitor renal function. We have assessed renal function by urinary protein excretion, fractional urinary excretion of sodium, and creatinine clearance. In particular, serum creatinine has been widely used as a GFR marker, although its limitations are well reported. ${ }^{35}$ It often overestimates GFR, misses some subclinical kidney dysfunction and changes slowly with age. It was recently reported that elevated circulating advanced glycation end products (AGEs) are an independent predictor of decline in renal function in older adults. ${ }^{36}$ Therefore, the use of markers which enable greater sensitivity to detect early changes in renal function might provide different results. 


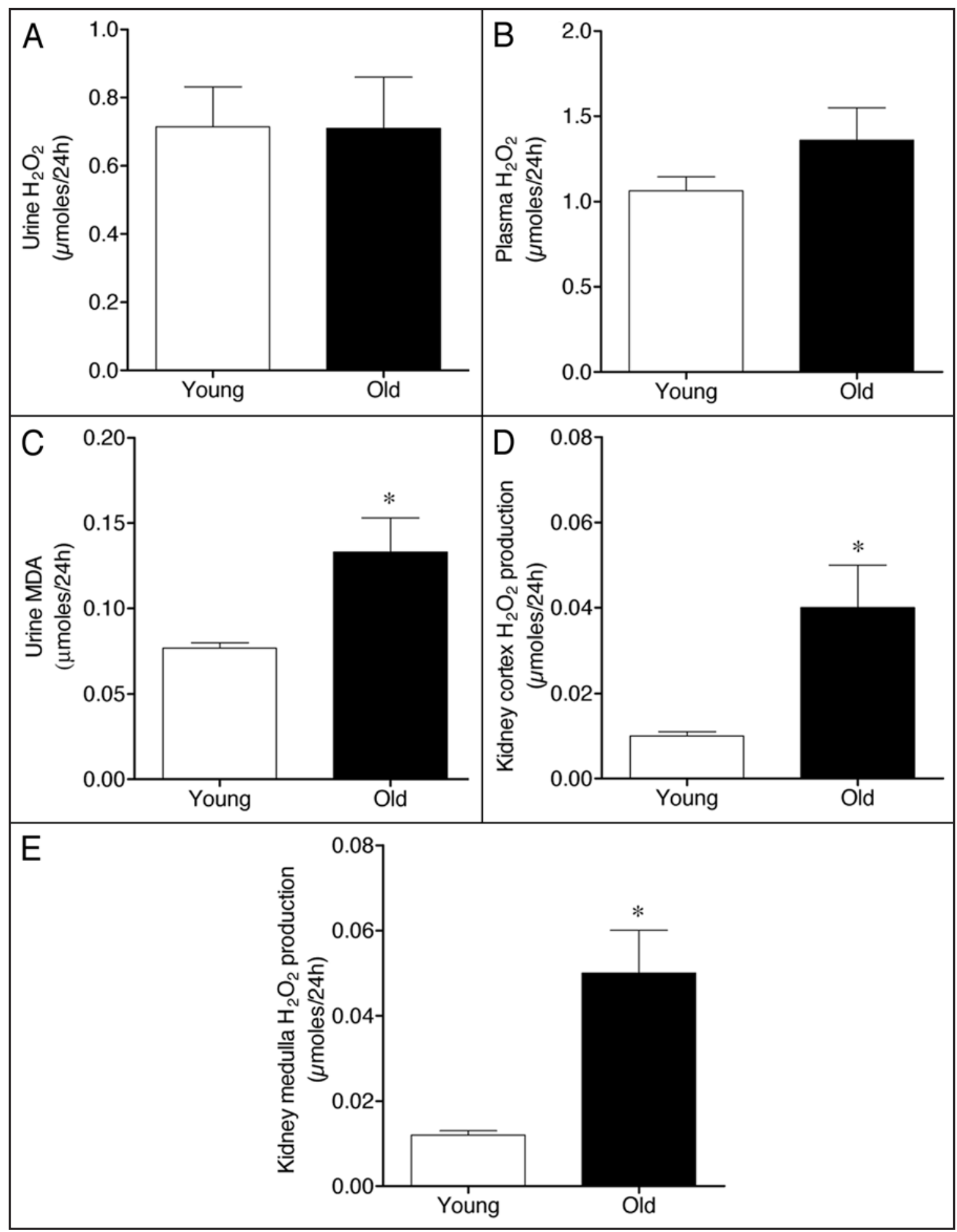

Figure 2. Hydrogen peroxide $\left(\mathrm{H}_{2} \mathrm{O}_{2}\right)$ levels in (A) urine and (B) plasma, (C) MDA content in urine, and the production of $\mathrm{H}_{2} \mathrm{O}_{2}$ by renal (D) cortex and $(E)$ medulla of young and old WKY rats. Each column represents the mean \pm SEM of six rats. Significantly different from values in young rats ( ${ }^{*} P$ $<0.05)$ using the Newman-Keuls test.

Previous studies have shown that oxidative stress is involved in the aging process and disease. ${ }^{19,20}$ Numerous changes in markers of oxidative stress have been documented in animals and humans at old age, including increased levels of $\mathrm{H}_{2} \mathrm{O}_{2}$ and MDA. ${ }^{12,14}$
Our results indicate that $\mathrm{H}_{2} \mathrm{O}_{2}$ concentration in plasma and urine, a commonly used biomarker of systemic oxidative stress, was not affected by aging in WKY. However, we can not rule out the possibility that this result may be due to biological 


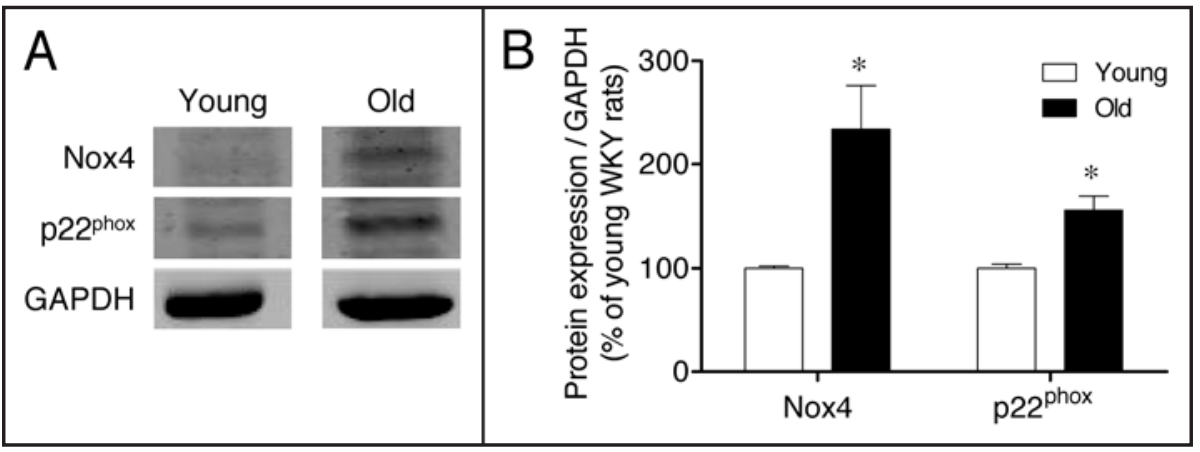

Figure 3. (A) Expression of the NADPH oxidase subunits Nox4 and $p 22^{\text {phox }}$ in the renal cortex of young and old WKY rats. GAPDH was used to control for loading differences. (B) The bar graph shows a summary of densitometric data. Values are normalized to the level of GAPDH expression in each condition and expressed as \% of young rats. Each column represents the mean \pm SEM of six independent immunoblots. Significantly different from values in young rats $\left({ }^{*} p<0.05\right)$ using the Newman-Keuls test.

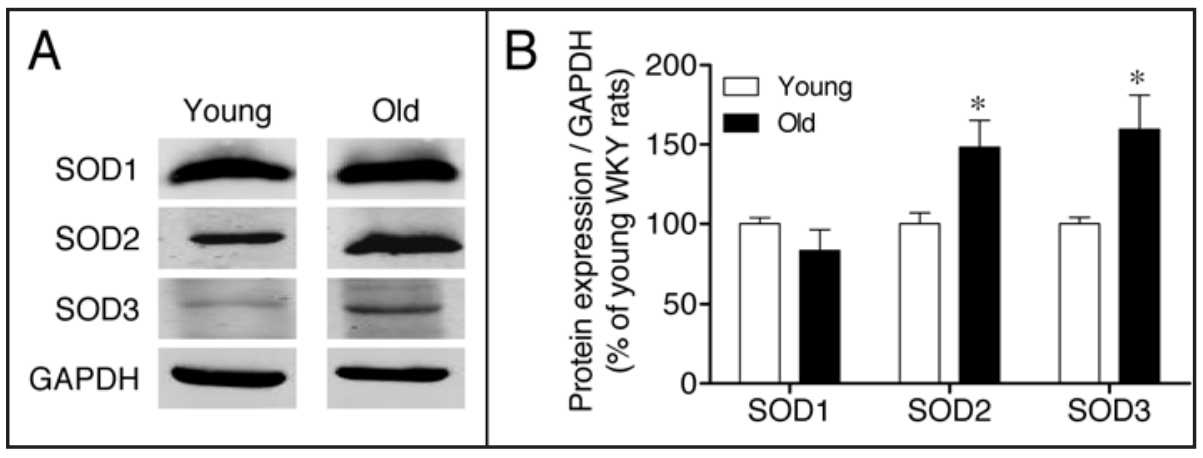

Figure 4. (A) Expression of superoxide dismutase isoenzymes SOD1, SOD2 and SOD3 in the renal cortex of young and old WKY rats. (B) The bar graph shows a summary of densitometric data. Values are normalized to the level of GAPDH expression in each condition and expressed as \% of young rats. Each column represents the mean \pm SEM of four independent immunoblots. Significantly different from values in young rats $\left({ }^{*} p<0.05\right)$ using the Newman-Keuls test.

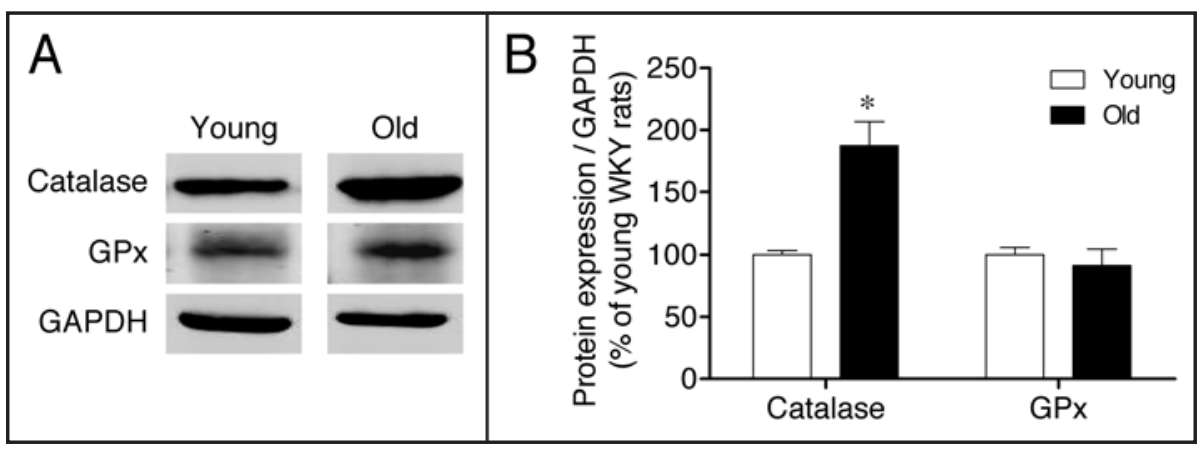

Figure 5. (A) Expression of catalase and glutathione peroxidase (GPx) in the renal cortex of young and old WKY rats. (B) The bar graph shows a summary of densitometric data. Values are normalized to the level of GAPDH expression in each condition and expressed as \% of young rats. Each column represents the mean \pm SEM of four independent immunoblots. Significantly different from values in young rats ( $\left.{ }^{*} p<0.05\right)$ using the Newman-Keuls test.

variation or instability of $\mathrm{H}_{2} \mathrm{O}_{2}$ in our samples. Because endproducts resulting from oxidative modifications inflicted on biomolecules are usually stable, we determined urinary MDA as a marker of lipid peroxidation. We found that old WKY had higher urinary excretion of MDA, which might reflect increased MDA in renal tissue. We therefore determined renal oxidative stress status by measuring the rate of $\mathrm{H}_{2} \mathrm{O}_{2}$ production by the kidney. It is reported here that renal cortical and medullary $\mathrm{H}_{2} \mathrm{O}_{2}$ production was markedly augmented in old WKY. This suggests that the dramatic increase in the rate of $\mathrm{H}_{2} \mathrm{O}_{2}$ production in kidneys of old WKY is indicative of a significant increase in oxidative stress in this tissue. Moreover, the increase in renal ROS production and associated oxidative damage to lipids may play a role in the pathogenesis of renal diseases. Altogether, these data are indicative that aged WKY presented first signs of renal oxidative damage.

In support of our results, others have shown that oxidative stress can lead to renal cell damage and impaired cellular function. ${ }^{37,38}$ Asghar and colleagues ${ }^{37}$ demonstrated that renal cells in culture from old rats are at a higher level of oxidative stress, which may contribute to the reduced dopamine $\mathrm{D}_{1}$ receptor function in cells from old compared with adult rats. A recent study showed that mitochondrial calpain 10 is required for cell viability and calpain 10 levels specifically decrease in aging rat, mice and human kidney tissues when renal function decreases, suggesting that calpain 10 is required for renal function and can serve as a biomarker of the aging kidney. ${ }^{38}$ Thus, it seems plausible that the enhancement of oxidative stress in the kidney of aged WKY could be injurious to renal function and structure.

This aspect is of crucial importance to understand the role of oxidative stress and alterations in renal function, which up to 52 weeks of age are not grossly apparent.

However, it should be underlined that subtle changes in receptor activation and signal transduction pathways have been associated with oxidative stress in renal proximal tubular cells in culture. These include the enhanced sensitivity to angiotensin II that correlates with the higher $\mathrm{H}_{2} \mathrm{O}_{2}$ generation and provokes an enhanced expression of glycosylated and non-glycosylated $\mathrm{AT}_{1}$ receptor forms in lipid rafts ${ }^{39}$ and the increased generation of $\mathrm{H}_{2} \mathrm{O}_{2}$, which may amplify the response downstream of $\alpha 1$-adrenoceptor activation. ${ }^{40}$ 
It is generally accepted that the age-related increases in ROS production and/or the diminished cellular antioxidant defense systems lead to an imbalance of prooxidant/antioxidant status. ${ }^{8,9}$ We report here the upregulation of the renal Nox4 and p22phox subunits of the oxidant-producing enzyme NADPH oxidase in aged WKY rats.

Following a similar trend, the expression of the antioxidant enzymes SOD2 and SOD3 isoforms was elevated in the kidneys of old rats. This may lead, at least in part, to enhanced renal $\mathrm{H}_{2} \mathrm{O}_{2}$ production observed in aged rats. Catalase and GPx are essential antioxidant enzymes since they are responsible for $\mathrm{H}_{2} \mathrm{O}_{2}$ detoxification. While expression of GPx remained unchanged during the aging process of WKY, expression of catalase was elevated in these rats. Renal overexpression of catalase may result from an adaptive response of old WKY to the excessive local production of reactive oxygen species. We hypothesize that the activation of antioxidant systems probably attenuates the increased levels of oxidative stress generated in this organ preventing the development of renal injury at the age of 52 weeks.

To the best of our knowledge, no studies evaluating the expression of oxidant and antioxidant enzymes in the kidney of young and aged WKY have been previously reported. Nevertheless, some studies have evaluated the expression and activity of oxidant and antioxidant enzymatic systems in other tissues and other rodent strains.

While some show age-dependent decreases in activity and/or expression, others show the opposite pattern. A recent study performed in the soleus muscle from young and aged WKY $\mathrm{WH}^{4}$ showed an increase in the activity and mRNA levels of SOD1, SOD2, catalase and GPx with aging, in parallel with an increase in tissue thiobarbituric acid reactive substances (TBARS) content, an indicator of lipid peroxidation. Furthermore, Judge and coworkers $^{42}$ recently reported age-associated increases in oxidative stress and SOD2, CAT and GPx activities in cardiac interfibrillar mitochondria from Fischer 344 rats. They suggested that the increases in antioxidant activities likely occurred in response to increased mitochondrial production of superoxide and $\mathrm{H}_{2} \mathrm{O}_{2}$. Therefore, the divergences observed in the literature are mainly due to differences in the type of animal, age and/or tissue used.

In conclusion, overexpression of renal NADPH oxidase subunits Nox4 and $2^{22^{p h o x}}$ and the resulting increases in renal $\mathrm{H}_{2} \mathrm{O}_{2}$ production in aged WKY does not associate with impairment in renal function or marked increases in blood pressure. It is suggested that increases in antioxidant defenses in aged WKY may correspond to an adaptive physiological response to counteract the age-associated deleterious effects resulting from increases in oxidative stress.

\section{Materials and Methods}

Animal preparation and experimental design. Five-week-old male Wistar Kyoto rats were obtained from Harlan-Interfauna Ibérica (Barcelona, Spain). Animals were housed under controlled conditions (12 hour light/dark cycle and room temperature at $22 \pm 2^{\circ} \mathrm{C}$ ) and had free access to tap water and standard rat chow (PANLAB, Barcelona, Spain). The animals were carefully maintained and monitored until 52 weeks of age. Blood pressure (systolic and diastolic) and heart rate were measured in conscious animals using a photoelectric tail-cuff detector (LE 5000, Letica, Barcelona, Spain). A minimum of five measures were made each time and the mean values were used for further calculations. Both weight and blood pressure measurements were performed at intervals of four to six weeks.

Metabolic study. 48 hours before experiments, young (13 weeks of age) and old ( 52 weeks of age) rats were placed in metabolic cages (Tecniplast, Buguggiate, Italy) for a 24-hour urine collection. The urine samples were collected in sterilized vials that were stored at $-80^{\circ} \mathrm{C}$ until assayed. After completion of this protocol, rats were anesthetized with sodium pentobarbital $(60 \mathrm{mg} / \mathrm{kg}$, i.p.). The animals were then sacrificed by exsanguination using cardiac puncture and the blood collected into tubes containing $\mathrm{K}_{3}$ EDTA for later determination of plasma biochemical parameters. Before excising their kidneys, the right ventricle of the heart was perfused with ice-cold saline $(0.9 \% \mathrm{NaCl})$ to remove all blood from the kidneys. The kidneys were then excised, weighed, decapsulated and the renal cortex and medulla rapidly separated by fine dissection. Tissue pieces were immediately frozen in liquid nitrogen and stored at $-80^{\circ} \mathrm{C}$ for western blot analysis.

Plasma and urine biochemistry. The quantification of sodium was performed by an ion-selective electrode. The analysis of non-fasting plasma creatinine, glucose, total cholesterol, urinary creatinine and urinary proteins was performed on the Cobas Mira Plus analyzer using standardized procedures (ABX Diagnostics for Cobas Mira, Switzerland). Creatinine clearance was calculated using $24 \mathrm{~h}$ urine creatinine excretion in absolute values $(\mathrm{ml} / \mathrm{min})$.

$\mathrm{H}_{2} \mathrm{O}_{2}$ concentration in urine and plasma. Hydrogen peroxide was measured fluorometrically using the Amplex Red Hydrogen Peroxide Assay kit (Molecular Probes Inc., Eugene, OR, USA). Amplex Red is a fluorogenic substrate with very low background fluorescence that reacts with $\mathrm{H}_{2} \mathrm{O}_{2}$ with a $1: 1$ stoichiometry to produce a highly fluorescent reagent. Measurement of $\mathrm{H}_{2} \mathrm{O}_{2}$ was evaluated using the last 24-h diluted (1:50) urine samples and plasma from young and old WKY rats. Fluorescence intensity was measured in a multiplate reader (Spectromax Gemini; Molecular Devices) at an excitation wavelength of $530 \mathrm{~nm}$ and emission wavelength of $590 \mathrm{~nm}$ at room temperature. After subtracting background fluorescence, the concentration of urinary $\mathrm{H}_{2} \mathrm{O}_{2}$ (in $\mu \mathrm{mol} / 24 \mathrm{~h}$ ) was calculated using a resorufin $-\mathrm{H}_{2} \mathrm{O}_{2}$ standard calibration curve generated from experiments using $\mathrm{H}_{2} \mathrm{O}_{2}$ and Amplex Red.

$\mathrm{H}_{2} \mathrm{O}_{2}$ production by renal cortex and medulla. Renal cortex and medulla were cut into square pieces and incubated at $37^{\circ} \mathrm{C}$ in Krebs-HEPES buffer (in mM: $\mathrm{NaCl} 118, \mathrm{KCl} 4.5, \mathrm{CaCl}_{2} 2.5$, $\mathrm{MgCl}_{2} 1.20, \mathrm{~K}_{2} \mathrm{HPO}_{4} 1.2, \mathrm{NaHCO}_{3}$ 25.0, Na-HEPES 25.0 and glucose 5; pH 7.4) for $90 \mathrm{~min}$. $\mathrm{H}_{2} \mathrm{O}_{2}$ released from the tissue was detected using the Amplex Red Hydrogen Peroxide Assay kit, as previously described. ${ }^{23}$

MDA determination. Briefly, urine samples were combined with $8.1 \%$ SDS for $10 \mathrm{~min}$. Equal volumes of 28\% TCA and $0.6 \%$ TBA were added and heated at $95^{\circ} \mathrm{C}$ during one hour. After cooling at room temperature, a mixture of chloroform/methanol 
(2:1) were added and centrifuged at 5,000 rpm for $10 \mathrm{~min}$. Supernatant absorbance was measured at $532 \mathrm{~nm}$.

The content of urinary MDA was calculated using a MDA standard calibration curve and results were expressed as micromoles of MDA per $24 \mathrm{~h}$ urine volume.

Western blotting. Renal cortices were lyzed in RIPA buffer containing $150 \mathrm{mM} \mathrm{NaCl}, 50 \mathrm{mM}$ Tris- $\mathrm{HCl}, \mathrm{pH}$ 7.4, $5 \mathrm{mM}$ EDTA, $1 \%$ Triton X-100, 0.5\% sodium deoxycholate, $0.1 \%$ SDS, $100 \mu \mathrm{g} / \mathrm{ml}$ PMSF, $2 \mu \mathrm{g} / \mathrm{ml}$ leupeptin and $2 \mu \mathrm{g} / \mathrm{ml}$ aprotinin. Protein concentration was determined using a protein assay kit (Bio-Rad Laboratories, Hercules, CA), with bovine serum albumin as standard. Cell lysates were boiled in 2x sample buffer $(62.5 \mathrm{mM}$ Tris- $\mathrm{HCl} \mathrm{pH} \mathrm{6.8,} \mathrm{2 \%} \mathrm{SDS,} \mathrm{10 \%} \mathrm{glycerol,} \mathrm{2 \%} 50$ mM DTT, $0.1 \% \mathrm{w} / \mathrm{v}$ bromophenol blue) at $95^{\circ} \mathrm{C}$ for five minutes. Samples containing $25-100 \mu \mathrm{g}$ of protein were separated by SDS-PAGE with $10 \%$ polyacrylamide gel and then electroblotted onto nitrocellulose membranes (Bio-Rad). Blots were blocked for one hour with 5\% non-fat dry milk in PBS $(10 \mathrm{mmol} / 1$ phosphatebuffered saline) at room temperature with constant shaking. Blots were then incubated with antibodies goat polyclonal anti-Nox4 (1:400, Santa Cruz Biotechnology); rabbit polyclonal anti-p22phox (1:800, Santa Cruz Biotechnology); rabbit polyclonal anti-SOD1 (1:2,000, Santa Cruz Biotechnology); goat polyclonal anti-SOD2 (1:100, Santa Cruz Biotechnology); goat polyclonal anti-SOD3 (1:500, Santa Cruz Biotechnology); rabbit polyclonal anti-catalase (1:2,000, Calbiochem); mouse monoclonal anti-glutathione peroxidase (1:1,000, Calbiochem) and mouse monoclonal antiGAPDH (1:60,000, Santa Cruz Biotechnology) in 5\% non-fat dry milk in PBS-T overnight at $4^{\circ} \mathrm{C}$. The immunoblots were subsequently washed and incubated with fluorescently labeled goat anti-rabbit (1:20,000; IRDyeTM 800, Rockland), fluorescently labeled donkey anti-goat (1:20,000; IRDyeTM 800, Rockland), or the fluorescently labeled goat anti-mouse secondary antibody (1:20,000; AlexaFluor 680, Molecular Probes) for $60 \mathrm{~min}$ at room temperature and protected from light. The membrane was washed and imaged by scanning at both 700 and $800 \mathrm{~nm}$, with an Odyssey Infrared Imaging System (LI-COR Biosciences).

Drugs. All chemicals were obtained from Sigma (St. Louis, MO) unless otherwise stated.

Data analysis. Geometric mean values are given with 95\% confidence limits and arithmetic means are given with SEM. Statistical analysis was performed by one-way analysis of variance (ANOVA) followed by Newman-Keuls test or Dunnett test for multiple comparisons. A p value less than 0.05 was assumed to denote a significant difference.

\section{Acknowledgements}

Supported by Fundação para a Ciência e a Tecnologia, FEDER and Programa Comunitário de Apoio (PIC/IC/83204/2007).

\section{References}

1. Clark B. Biology of renal aging in humans. Adv Ren Replace Ther 2000; 7:11-21.

2. Davies I, Fotheringham AP, Faragher BE. Age-associated changes in the kidney of the laboratory mouse. Age Ageing 1989; 18:127-33.

3. Epstein M. Aging and the kidney. J Am Soc Nephrol 1996; 7:1106-22.

4. Hirokawa K. Characterization of age-associated kidney disease in Wistar rats. Mech Ageing Dev 1975; 4:301-16.
5. Martin JE, Sheaff MT. Renal ageing. J Pathol 2007; 211:198-205.

6. Zhou XJ, Rakheja D, Yu X, Saxena R, Vaziri ND, Silva FG. The aging kidney. Kidney Int 2008; 74:710-20.

7. Johnson FB, Sinclair DA, Guarente L. Molecular biology of aging. Cell 1999; 96:291-302.

8. Harman D. Aging: A theory based on free radical and radiation chemistry. J Gerontol 1956; 11:298-300.

9. Harman D. Aging and oxidative stress. J Int Fed Clin Chem 1998; 10:24-7.

10. Beckman KB, Ames BN. The free radical theory of aging matures. Physiol Rev 1998; 78:547-81.

11. Hamilton ML, Van Remmen H, Drake JA, Yang H, Guo ZM, Kewitt K, et al. Does oxidative damage to DNA increase with age? Proc Natl Acad Sci USA 2001; 98:10469-74.

12. Ku HH, Brunk UT, Sohal RS. Relationship between mitochondrial superoxide and hydrogen peroxide production and longevity of mammalian species. Free Radic Biol Med 1993; 15:621-7.

13. Zhou XJ, Vaziri ND, Zhang J, Wang HW, Wang XQ. Association of renal injury with nitric oxide deficiency in aged SHR: Prevention by hypertension control with AT1 blockade. Kidney Int 2002; 62:914-21.

14. Mooradian AD, Lung CC, Shah G, Mahmoud S, Pinnas JL. Age-related changes in tissue content of malondialdehyde-modified proteins. Life Sci 1994; 55:1561-6.

15. Rabbani N, Sebekova K, Sebekova K Jr, Heidland A, Thornalley PJ. Accumulation of free adduct glycation, oxidation and nitration products follows acute loss of renal function. Kidney Int 2007; 72:1113-21.

16. Brown-Borg HM, Rakoczy SG. Catalase expression in delayed and premature aging mouse models. Exp Gerontol 2000; 35:199-212.

17. Tarry-Adkins JL, Joles JA, Chen JH, Martin-Gronert MS, van der Giezen DM, Goldschmeding R, et al. Protein restriction in lactation confers nephroprotective effects in the male rat and is associated with increased antioxidant expression. Am J Physiol Regul Integr Comp Physiol 2007; 293:1259-66.

18. Tarry-Adkins JL, Ozanne SE, Norden A, Cherif H, Hales CN. Lower antioxidant capacity and elevated p 53 and p 21 may be a link between gender disparity in renal telomere shortening, albuminuria and longevity. Am J Physiol Renal Physiol 2006; 290:509-16.

19. Bokov A, Chaudhuri A, Richardson A. The role of oxidative damage and stress in aging. Mech Ageing Dev 2004; 125:811-26.

20. Kregel KC, Zhang HJ. An integrated view of oxidative stress in aging: Basic mechanisms, functional effects and pathological considerations. Am J Physiol Regul Integr Comp Physiol 2007; 292:18-36.

21. Hermanns RC, de Zwart LL, Salemink PJ, Commandeur JN, Vermeulen NP, Meerman JH Urinary excretion of biomarkers of oxidative kidney damage induced by ferric nitrilotriacetate. Toxicol Sci 1998; 43:241-9.

22. Caldeira da Silva CC, Cerqueira FM, Barbosa LF, Medeiros MH, Kowaltowski AJ. Mild mitochondrial uncoupling in mice affects energy metabolism, redox balance and longevity. Aging Cell 2008; 7:552-60.

23. Furukawa S, Fujita T, Shimabukuro M, Iwaki M, Yamada Y, Nakajima Y, et al. Increased oxidative stress in obesity and its impact on metabolic syndrome. J Clin Invest 2004; 114:1752-61.

24. Kagota S, Yamaguchi Y, Tanaka N, Kubota Y, Kobayashi K, Nejime N, et al. Disturbances in nitric oxide/cyclic guanosine monophosphate system in SHR/NDmcrcp rats, a model of metabolic syndrome. Life Sci 2006; 78:1187-96.

25. Linz W, Jessen T, Becker RH, Scholkens BA, Wiemer G. Long-term ACE inhibition doubles lifespan of hypertensive rats. Circulation 1997; 96:3164-72.

26. Natalucci S, Ruggeri P, Cogo CE, Picchio V, Brunori A, Burattini R. Agerelated analysis of glucose metabolism in spontaneously hypertensive and normotensive rats. Exp Physiol 2003; 88:399-404.

27. Percy CJ, Brown L, Power DA, Johnson DW, Gobe GC. Obesity and hypertension have differing oxidant handling molecular pathways in age-related chronic kidney disease. Mech Ageing Dev 2009; 130:129-38.

28. Perrin D, Soulage C, Pequignot JM, Geloen A. Resistance to obesity in Lou/C rats prevents ageing-associated metabolic alterations. Diabetologia 2003; 46:1489-96.

29. Susic D, Varagic J, Frohlich ED. Isolated systolic hypertension in elderly WKY is reversed with L-arginine and ACE inhibition. Hypertension 2001; 38:1422-6.

30. Franklin SS, Jacobs MJ, Wong ND, L'Italien GJ, Lapuerta P. Predominance of isolated systolic hypertension among middle-aged and elderly US hypertensives: Analysis based on National Health and Nutrition Examination Survey (NHANES) III. Hypertension 2001; 37:869-74.

31. Antikainen R, Jousilahti P, Tuomilehto J. Systolic blood pressure, isolated systolic hypertension and risk of coronary heart disease, strokes, cardiovascular disease and all-cause mortality in the middle-aged population. J Hypertens 1998; 16:577-83.

32. Qureshi AI, Suri MF, Mohammad Y, Guterman LR, Hopkins LN. Isolated and borderline isolated systolic hypertension relative to long-term risk and type of stroke: A 20-year follow-up of the national health and nutrition survey. Stroke 2002; 33:2781-8.

33. Stern JS, Gades MD, Wheeldon CM, Borchers AT. Calorie restriction in obesity: Prevention of kidney disease in rodents. J Nutr 2001; 131:913-7. 
34. Li YM, Steffes M, Donnelly T, Liu C, Fuh H, Basgen J, et al. Prevention of cardiovascular and renal pathology of aging by the advanced glycation inhibitor aminoguanidine. Proc Natl Acad Sci USA 1996; 93:3902-7.

35. Giles PD, Fitzmaurice DA. Formula estimation of glomerular filtration rate: Have we gone wrong? BMJ 2007; 334:1198-200.

36. Semba RD, Fink JC, Sun K, Bandinelli S, Guralnik JM, Ferrucci L. Carboxymethyllysine, an advanced glycation end product and decline of renal function in older community-dwelling adults. Eur J Nutr 2009; 48:38-44

37. Asghar M, Chillar A, Lokhandwala MF. Renal proximal tubules from old Fischer 344 rats grow into epithelial cells in cultures and exhibit increased oxidative stress and reduced D1 receptor function. Am J Physiol Cell Physiol 2008; 295:1326-31.

38. Covington MD, Arrington DD, Schnellmann RG. Calpain 10 is required for cell viability and is decreased in the aging kidney. Am J Physiol Renal Physiol 2009; 296:478-86.

39. Pedrosa R, Villar VA, Pascua AM, Simao S, Hopfer U, Jose PA, Soares-da-Silva P. $\mathrm{H}_{2} \mathrm{O}_{2}$ stimulation of the $\mathrm{Cl}^{-} / \mathrm{HCO}^{-}$exchanger by angiotensin II and angiotensin II type 1 receptor distribution in membrane microdomains. Hypertension 2008; 51:1332-8.

40. Simao S, Fraga S, Jose PA, Soares-da-Silva P. Oxidative stress and $\alpha 1$-adrenoceptormediated stimulation of the $\mathrm{Cl}^{-} / \mathrm{HCO}^{-}$exchanger in immortalized SHR proximal tubular epithelial cells. Br J Pharmacol 2008; 153:1445-55.

41. Lambertucci RH, Levada-Pires AC, Rossoni LV, Curi R, Pithon-Curi TC. Effects of aerobic exercise training on antioxidant enzyme activities and mRNA levels in soleus muscle from young and aged rats. Mech Ageing Dev 2007; 128:267-75.

42. Judge S, Jang YM, Smith A, Hagen T, Leeuwenburgh C. Age-associated increases in oxidative stress and antioxidant enzyme activities in cardiac interfibrillar mitochondria: implications for the mitochondrial theory of aging. FASEB J 2005; 19:419-21. 


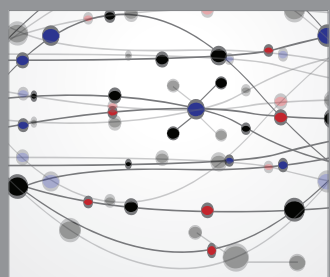

The Scientific World Journal
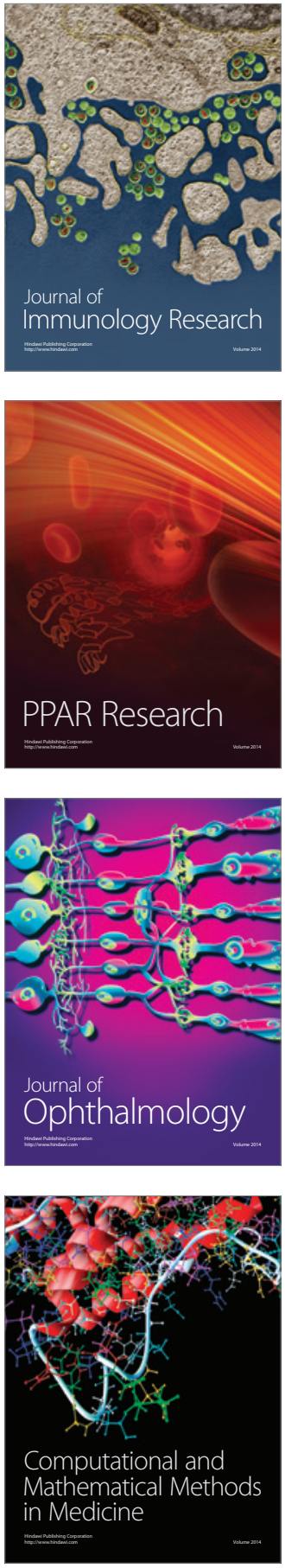

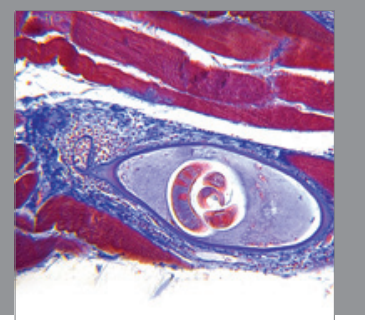

Gastroenterology

Research and Practice
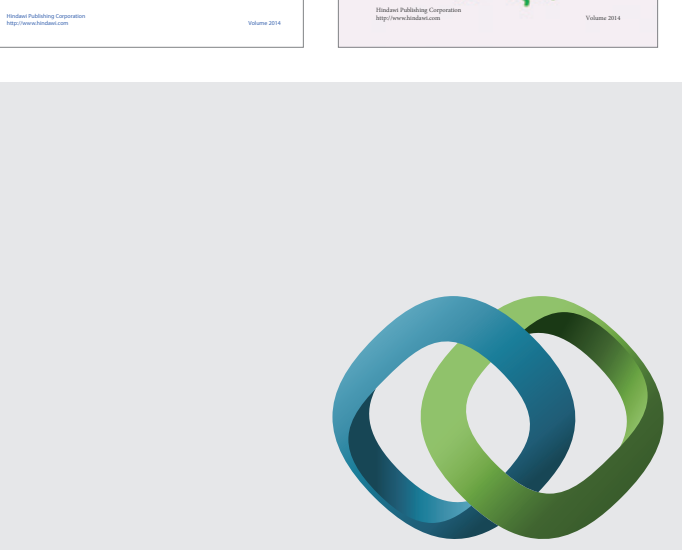

\section{Hindawi}

Submit your manuscripts at

http://www.hindawi.com
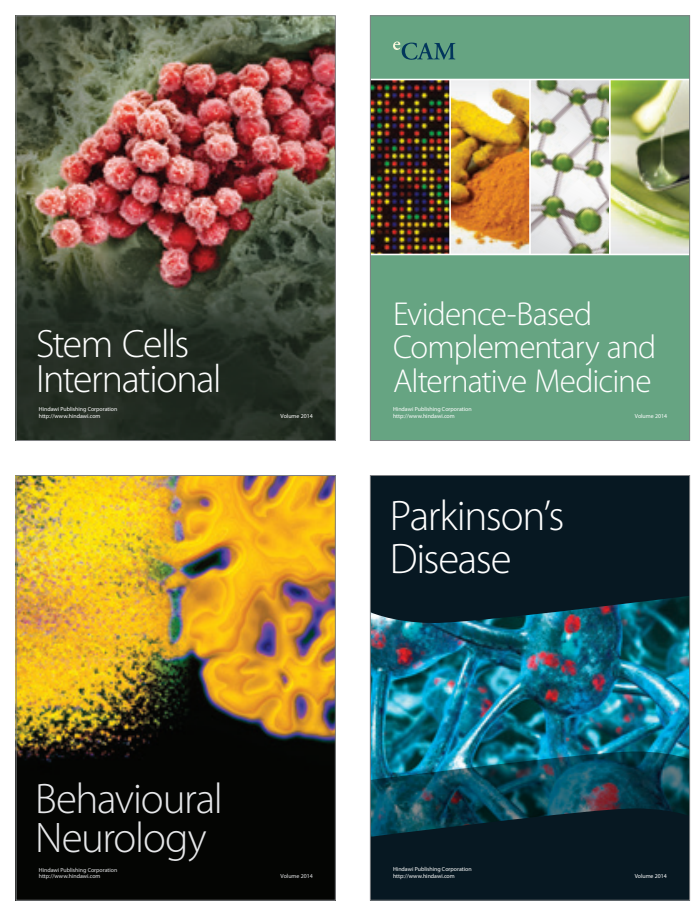

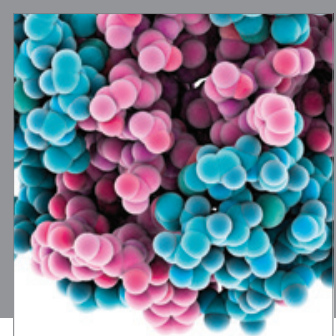

Journal of
Diabetes Research

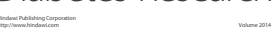

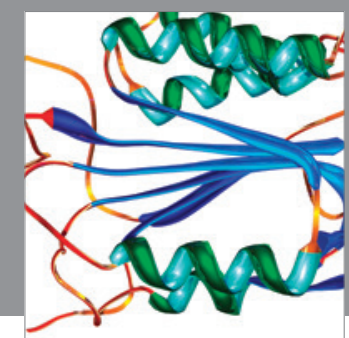

Disease Markers
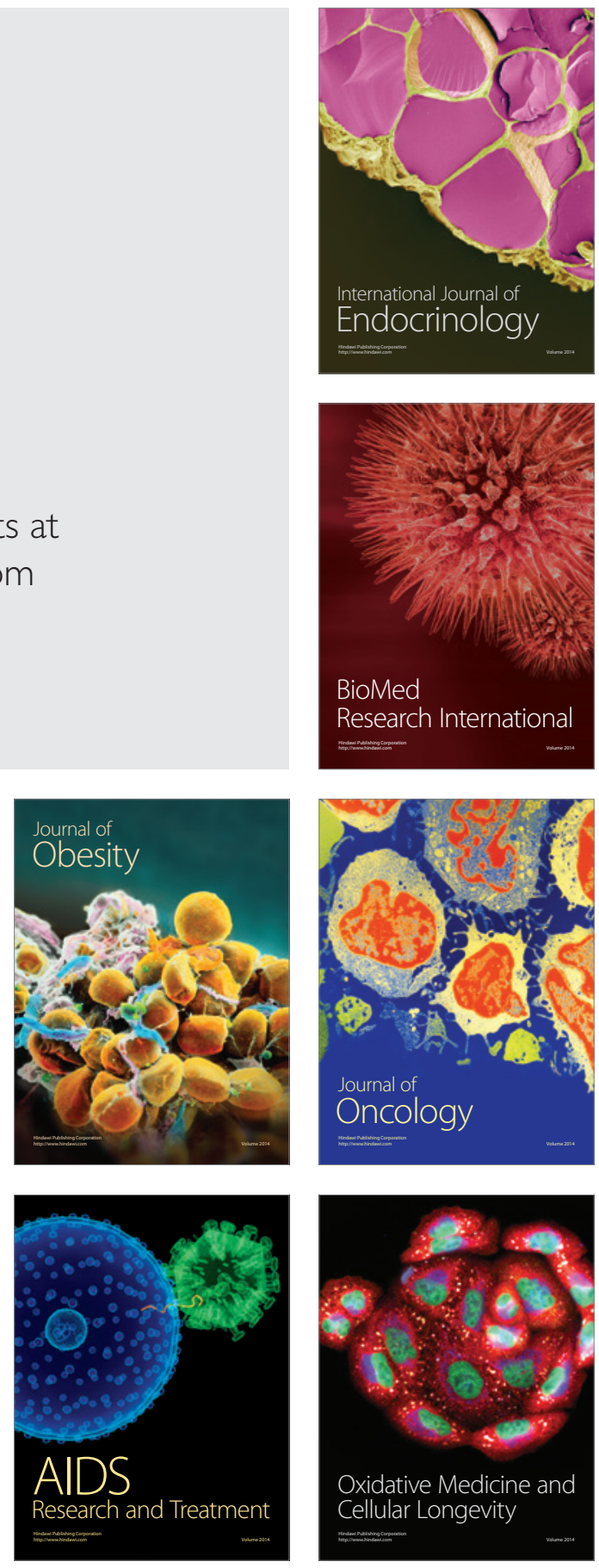